Journal of Applied Fluid Mechanics, Vol. 15, No. 1, pp. 231-243, 2022.

Available online at www.jafmonline.net, ISSN 1735-3572, EISSN 1735-3645.

https://doi.org/10.47176/jafm.15.01.32835

\title{
Effects of Blowing Location on Aeroacoustics of the Flow over a Circular Cylinder
}

\author{
S. Abbasi \\ Arak University of Technology, Arak, Markazi, 38181-41167, Iran \\ Email: s_abbasi@arakut.ac.ir
}

(Received March 30, 2021; accepted August 14, 2021)

\begin{abstract}
This study investigated the effect of blowing on vortex shedding and aerodynamic noise due to flow over a circular cylinder. The flow simulation is performed by the URANS equations using k- $\omega$-SST turbulence model. Calculations of the aerodynamic noise are performed through F-fowcs Williams-Hawkins analogy. The cylinder's cross-section with a diameter of $\mathrm{D}=16 \mathrm{~mm}$, and the blowing jet is applied through slot windows that are located on the cylinder back surface. In this study, three positions for slot windows are considered. Verification of the numerical results is confirmed by comparing numerical results with the reported experimental ones (Uncontrolled case). The results showed that the optimal blowing position could reduce noise, lift coefficient, and drag coefficient; this result occurs for a slot window located near the cylinder horizontal axis of symmetry. On the other hand, if the blowing slot is located at the cylinder symmetric vertical axis, the aerodynamic noise increases by approximately $4 \mathrm{~dB}$. In this case, the average lift and drag fluctuations are increased by more than $200 \%$. The present study gives a new idea to the reduction of noise of the single body systems.
\end{abstract}

Keywords: Circular cylinder; Reduction noise; Aerodynamic noise; Vortex shedding.

\section{Introduction}

Interaction of a uniform flow with a bluff body produces periodic aerodynamic forces, which can cause highly nonlinear vibrations and excessive noise. The interaction of flow with the bluff body such as circular cylinder and square cylinder due to their application in the industry (heat exchangers, cables, bridges, high rise buildings, transmission lines, and aircraft landing gear systems) has been the focus of many researchers (Bao et al. 2018, Ma et al. 2019, Zhu et al. 2019a; 2019b, Abbasi and Souri 2021a,b and Souri and Mojra 2021). The flow around the circular cylinders shows a significant flow pattern that has been the subject of much experimental and numerical research. Norberg (1987) observed that fluid flow is detached from the surface of the object which results in creating vortices that are started to move towards downstream flow. In an experimental study, Schlichting and Gersten (2016) predicted the Strouhal number based on the cylinder diameter and freestream speed equal to 0.2. Aradag et al. (2011) predicted the drag coefficient and the Strouhal number to be 1.2 and 0.2 , respectively, in a Three-Dimensional (3D) Turbulent Flow Behind a Circular Cylinder. Seo and Moon (2007) simulated the aerodynamic noise emitted from a circular cylinder using the LES model. Gao et al. (2019) and Chen et al. (2020) studied the flow around three-circular cylinders due to three- circular cylinders widespread use, especially the chimney group and chemical reaction towers. They found that when the distance between the cylinders was three times that of diameter, the fluctuations of the aerodynamic forces increased dramatically, and consequently, the pressure field was affected.

The flow structure around a body is mainly investigated by finding attractive and efficient control methods. The control method can be used active or passive, depending on its application. Among the passive methods recently studied by Zhu et al. (2019b) was the use of fin-shaped appendages on the front surface of the symmetrical cylinder to control flow and separation angles. They found that when the fins were placed at $\boldsymbol{\theta} \leq \mathbf{4 0}$ and $\boldsymbol{\theta} \geq \mathbf{7 0}$, the length of the wake became shorter, while between $50^{\circ}$ and $\mathbf{6 0}^{\circ}$ degrees, the wake became wider, resulting in rapid formation of vortex shedding and drag increases. Arcondoulis et al. (2021) investigated the porous coating on the surface of a circular cylinder as an experimental model. They found that the leeward side porosity strongly influences the drag coefficient. Zhang et al. (2020) investigated the effect of the non-uniform porous coating over the circumference of the circular 
cylinder on aerodynamic noise as a numerical model. Their numerical results show that applying highporosity material in the separation zone is most effective on reducing aerodynamic drag and noise. Other properties and structures of the porous coating on aerodynamic noise have also been investigated by researchers (Arcondoulis et al. 2019 and Li et al. 2020).

The most significant advantage of active methods over passive methods is the control of their performance over time. Unfortunately, the cost of using active methods is much higher than inactive ones (Zhu et al. 2020). Recently, Assi et al. (2019) Used eight small rotating cylinders with a diameter of $0.05 \mathrm{D}$ which were surrounded by a circular cylinder of diameter D, to control the flow and the suppression of vortex shedding. They found that if the speed of rotation of the control cylinders was equal to $\boldsymbol{\omega}=\frac{6 U}{r}$ ( $\boldsymbol{U}$ : the free stream velocity, $r$ : Control cylinder radius), the drag coefficient would be significantly reduced. Souri and Mojra (2021) achieved a combination of active and passive methods to reduce the aerodynamic noise caused by a circular cylinder. Among the active methods of flow control, the subject of blowing current is a favorite of many researchers. Various studies have been conducted in this field, but little research has been done on the effects of using this method on the aerodynamic noise properties. For example, Abbasi and Souri used simultaneous suction and blowing to reduce aerodynamic noise in a rod - airfoil configuration (Abbasi and Souri 2020, Abbasi and Souri 2021a). Mathelin et al. (2002) experimentally examined the heat transfer around a cylinder containing blowing slot windows. In this study, the blowing mass flow rate was continuous at Reynolds numbers ranging from 3900 to 14000 , and the injection rate was considered variable. They observed that the boundary layer thickness increases when the blowing injection rate is increased; a 5\% increase in the injection rate, doubles the boundary layer thickness and reduces friction and drag as well. Blowing can be used as an actuator that controls vortex shedding and reduces drag force acting on the cylinder. Chen et al. (2020) studied a passive flow control method experimentally by employing windward suction combined with a leeward jet over a circular cylinder for drag reduction and dynamic wind loading suppression. The results showed that the periodicity of the vortex shedding was diminished and eventually disappeared with more suction/jet holes. Zhdanov et al. (2001) investigated the effect of locating a blowing slot with a length of $10 \%$ of the cylinder diameter at different positions on the cylinder surface and a blowing speed of $65 \%$ of the freestream velocity; the Reynolds number was 15,000 . Their results showed a reduction in the drag coefficient at the optimum slot location. By comparing the studies by Mathelin et al. (2002) and Zhdanov et al. (2001), it can be observed that the slot location has an important role in the flow structure and drag reduction. Schetz and Nerney (1977) observed that the increase in the injection mass flow rate, velocity and turbulence intensity in the boundary layer was increased. Feng and Wang
(2010) and Feng et al. (2011) carried out experimental studies on the role of a jet in controlling the vortex shedding in the cylinder wake. Their results revealed that the vortex pair created by the jet had a significant effect on the flow behind the circular cylinder. Williams et al. (1992) and Lin et al. (1995) pinpointed that a favorable suction or blowing, applied through very small holes provided in a helical pattern on the surface of the cylinder, would reduce or eliminate the vortex shedding. Inoue et al. (2003) investigated control of Aeolian tones radiated from a circular cylinder using blowing/suction.

A review of the literature reveals that most of the studies focus on the flow structure of circular cylinders and research on aerodynamic noise is scarce. Hence, further research on the impact of aerodynamic noise active control methods for circular cylinders is necessary to supplement the literature. In the present study, the effect of air blowing location on the aerodynamic noise control in a circular cylinder is investigated. This study addresses and evaluates the unsteady flow structure, vortex shedding and the effect of the active control method on reducing or increasing the generated sound, and founded the relation between the blowing location and generated sound level is identified.

\section{MeTHODOLOGY}

In the present study, FW-H analog (Williams and Hawkings 1969) is used to predict aerodynamic noise, but it is necessary to extract the flow characteristics to be solved as the input of the equations governing the acoustics. For this purpose, continuity (Eq. 1) and momentum (Eq. 2) equations for incompressible flow are defined as follows (Samion et al. 2016):

$$
\begin{aligned}
& \frac{\partial u_{\mathrm{i}}}{\partial \mathrm{x}_{\mathrm{i}}}=\mathbf{0} \\
& \frac{\partial\left(\mathrm{u}_{\mathrm{i}}\right)}{\partial \mathrm{t}}=-\frac{1}{\rho} \frac{\partial \mathrm{p}}{\partial \mathrm{x}_{\mathrm{i}}}+v \frac{\partial^{2} u_{i}}{\partial x_{i} \partial x_{j}}-\frac{\partial}{\partial \mathrm{x}_{\mathrm{i}}}\left(\boldsymbol{u}_{\mathrm{i}} u_{\mathrm{j}}-\overline{\mathbf{u}_{{ }_{\imath}{ }_{\imath} \mathbf{u}_{\mathrm{j}}^{\prime}}}\right)
\end{aligned}
$$

In the above relations $\rho, u$, and $p$ are the density, the fluid velocity, and static pressure, respectively. Where $x_{i}$ and $x_{j}$ indicate the direction of flow and the direction perpendicular to the flow, respectively. $u_{\mathrm{i}}$ and $u_{\mathrm{j}}$ are the corresponding time-averaged velocity components and $u_{i}^{\prime}$ depicts the fluctuating part of the velocity and $\left(\overline{\mathrm{u}^{\prime}{ }_{1} \mathrm{u}_{\mathrm{j}}^{\prime}}\right)$ is the Reynolds stress.

To predict aeroacoustics characteristics, aside from the Navier-Stokes equations, the Williams-Hawkins equation (FW-H) (Eq. 3) (Williams and Hawkings 1969) is used as well. This analogy considered the effect of the optional motion of the sound source. This is a heterogeneous wave equation derived from the continuity equation and Navier-Stokes equations.

$$
\frac{1}{c_{0}^{2}} \frac{\partial^{2} p^{\prime}}{\partial t^{2}}-\nabla^{2} p^{\prime}=\underbrace{\frac{\partial}{\partial t}\left(\left(\rho_{0} v_{n}+\rho\left(u_{n}-v_{n}\right)\right) \delta(f)\right)}_{\text {monopole }}
$$




$$
\begin{array}{r}
-\underbrace{\frac{\partial}{\partial x_{i}}\left(\left(P_{i j} n_{j}+\rho u_{i}\left(u_{n}-v_{n}\right) \delta(f)\right)\right.}_{\text {dipole }} \\
+\underbrace{\frac{\partial^{2}}{\partial x_{i} \partial x_{j}}\left(T_{i j} H(f)\right)}_{\text {quadrupole }}
\end{array}
$$

Where $c_{0}$ denotes the speed of sound in a far field and $u_{i}$ is the fluid velocity in the $x_{i}$ direction. $H(f)$ is the Heaviside function and $\delta(f)$ denotes the DIRAC Delta function. $u_{n}$ and $v_{n}$ are the fluid velocity in the direction normal to the integration surface and the surface velocity in the direction normal to the integration surface, respectively. $\rho$ is the fluid density where the suffix zero refers to atmospheric values. Furthermore, $\tau_{i j}, \delta_{i j}, \rho$ and $P^{\prime}$ are respectively the viscous stress, Kronecker delta, the density and the sound pressure in the farfield in $p^{\prime}=p-p_{0}$.

The first term of the right of the Eq. 3 refers to the monopole sound source, also known as the thickness noise, which is caused by fluid displacement by the body surface in the flow field. The second term, which is related to the forces applied to the surface of the body, is known as the dipole or loading source. In Eq. $3, \delta(f)$ represents the mono-and dipole sources. The last term of the right of Eq. 3 is for quadrupole sources, which is caused by unsteady shear stresses and these are outside the source surface and have the lowest contribution in each period of sound generation so it can be neglected. The quadrupole term is the distribution of the volume of sources defined by the Heaviside function $H(f)$. In FW-H equation, $f=0$ indicates the source surface, $f>0$ is unbounded space, $n_{i}$ represents the perpendicular unit vector towards outside the area, and $P_{i j}$ is the compressive stress tensor.

To solve the FW-H equation, the free-space Green's function is utilized to compute the sound pressure at the receiver location $x$. Since quadrupole sources do not effectively play a role in noise generation for Mach number, it is ignored in Eq. 4, which shows that the pressure is acoustic and is derived from Eq. 3 .

$$
\begin{aligned}
& p^{\prime}(x . t) \\
& =\frac{1}{4 \pi}\left(\left(\frac{\partial}{\partial t}\right) \int_{S}\left[\frac{\rho_{0} n_{i}\left(\left(1-\frac{\rho}{\rho_{0}}\right) v_{i}+\frac{\rho u_{i}}{\rho_{0}}\right)}{(r(1-M))}\right]_{r e t}\right) \\
& +\underbrace{\frac{1}{4 \pi}\left(\left(-\frac{\partial}{\partial x_{i}}\right) \int_{S}\left[\frac{P_{i j} n_{i}+\rho u_{i}\left(u_{i_{n}}-u_{j_{n}}\right)}{(r(1-M))}\right]_{r e t} d S\right)}_{\text {monopole term }}
\end{aligned}
$$

Here the subscript $\left(r_{e t}\right)$ means for the retarded time, and $\mathrm{M}$ is local Mach number.

In the current study, numerical analysis of the aerodynamic noise generated by the transient flow over a circular cylinder and the noise reduction are examined. To this aim, a cylinder with a circular cross-section in which diameter length is $16 \mathrm{~mm}$, is considered and a flow velocity of $20 \mathrm{~m} / \mathrm{s}$ is assumed. The conditions for the cylinder in this study are similar to those of the Caselino and Jacob model (Casalino and Jacob 2003) that was an experimental study on the circular cylinders. The Reynolds and Mach numbers are $2.2 \times 10^{4}$ and 0.06 , respectively. Due to the reduction of computational costs, the $2 \mathrm{D}$ URANS model has been used. To record the sound generated by the transient flow over the cylinder, a microphone distant $1.38 \mathrm{~m}$ from the top of the cylinder and in the direction perpendicular to the cylinder center is employed. A schematic diagram of the computational domain, boundary conditions, and the microphone location is shown in Fig. 1.

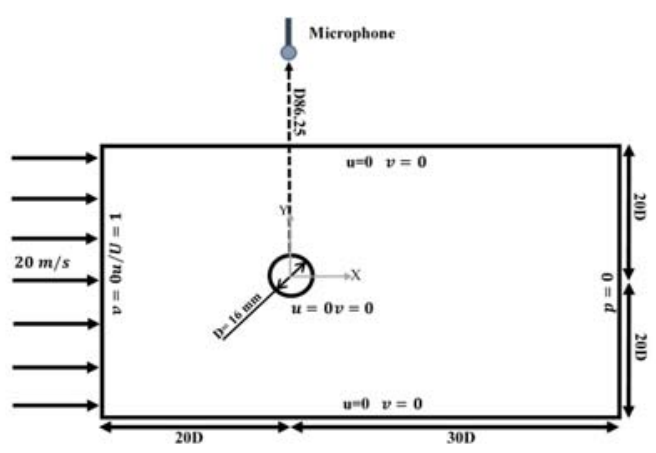

Fig.1. Schematic diagram of the computational domain and the boundary conditions.

In the present study, numerical analysis of the flow was performed using the Ansys Fluent software. The exact time-accurate solution is performed using the Unsteady Rynolds Averaged Navier-Stokes equations (URANS). The k- $\omega$-sst turbulence model has been used to accurately estimate viscosity eddies. Stability, economy, and appropriate precision for a wide range of turbulent flows confirm its popularity in the simulation of turbulent heat transfer and flow in the industry (Rocha et al. 2014, Zhang et al. 2016, Chong et al. 2017). At the inlet boundary, a uniform flow with $20 \mathrm{~m} / \mathrm{s}$ is considered, and at the outlet boundary, the static pressure (relative to the ambient pressure) of the flow is assumed to be zero. On the upper and lower boundaries, the no-slip condition has been applied. The Simple algorithm is used to couple the velocity field with the pressure field. For the spatial discretization of the governing equations, a second-order upwind scheme is employed for the discretization of momentum, turbulent kinetic energy, and specific dissipation rate, while the Pressure-Staggering Option (PRESTO) scheme is used for the discretization of pressure. Additionally, For the temporal discretization, a second-order implicit scheme is used. In the unsteady simulation process, a time step of $0.0001 \mathrm{~s}$ was used and the total simulation time was $0.5 \mathrm{~s}$. The CFL (CourantFriedrichs-Lewy) number varied between 1 and 4 . 30 inner iterations were performed at each time interval. The condition for convergence in all 
governing equations implies that the residues must reach $10^{-7}$. Moreover, for the $\mathrm{FW}-\mathrm{H}$ results, a sampling frequency equal $10 \mathrm{kHz}$ is employed. The source correlation length for the present study based on the uncontrolled cylinder, is considered $15 D$ in uncontrolled case (Table 1).

Table. 1. Dependence of predicted NSP on specified source correlation lengths (based on microphone 1).

\begin{tabular}{|c|c|c|c|c|c|}
\hline$L_{c}$ & $3 \mathrm{D}$ & $5 \mathrm{D}$ & $10 \mathrm{D}$ & $15 \mathrm{D}$ & $20 \mathrm{D}$ \\
\hline$S P L_{p}(\mathrm{~dB})$ & 57 & 64 & 72 & 74 & 74 \\
\hline
\end{tabular}

$L_{c}$; The source correlation length, D; Cylinder diameter, NSP; Noise spectra peak

Accurate estimation of flow disturbances is important. To accurately estimate severe gradients, meshing should be standardized to avoid possible mutations. Figure $2 \mathrm{~A}$ shows an open view of the solution field meshing, which is shown to be more efficient as it approaches the bluff body. The enlarged concentric square around the circular cylinder is discretized with 240 nodes, and the grid in the radial direction is denser near the cylinder surface (Fig. 2B). The distance of the nearest grid (the first mesh layer) in the fluid zone from the cylinder surface is $0.028 \mathrm{D}$, which plays an important role in estimating the flow behavior in the boundary layer. The $y^{+}$values are also considered as the best mesh for simulation. In fact, $y^{+}$denotes the nondimensionalized distance of the grid points from the wall that its value is lower than 1 .

To verify the mesh, its independence from the number of cells is evaluated. For this purpose, Meshing has been done for the various number of cells: 40000, 50000, 70000, 80000, and 90000. In each case, the obtained sound pressure level and the normalized velocity in the wake region have been considered as the most important results. In Fig. 3a, the SPL-St diagram of the microphone is shown for the different numbers of cells. The Strouhal number and SPL are defined in Eqs. 5,6. It is observed that increasing the number of cells from 40000 to 80000 causes significant changes in the sound pressure level. However, when the number of cells varies from 80000 to 90000 , no considerable change is observed in the results. A similar result can be seen in Fig. 3B. So, the number of 80000 cells have been selected for the present numerical analysis.

$$
\begin{aligned}
& S t=\frac{f D}{U} \\
& S P L(d B)=20 \log \left(\frac{P_{r m s}}{P_{\text {ref }}}\right)
\end{aligned}
$$

Here, $f, D$, and $U$ are are frequency, cylinder diameter, and freestream velocity, respectively. In Eq. 6, $P_{r m s}$ is the root mean square sound pressure and $P_{\text {ref }}$ is the reference sound pressure.

A unit circular cylinder with diameter $D$ is tested for validation. Besides, the numerical prediction of the noise emitted, the hydrodynamic parameters are

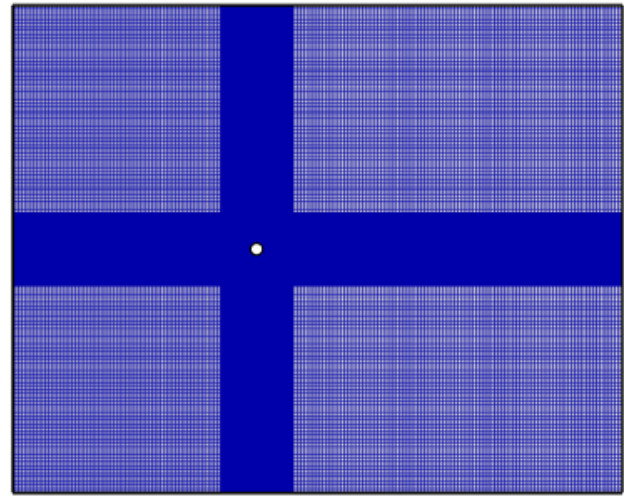

(A) full computational domain mesh structure

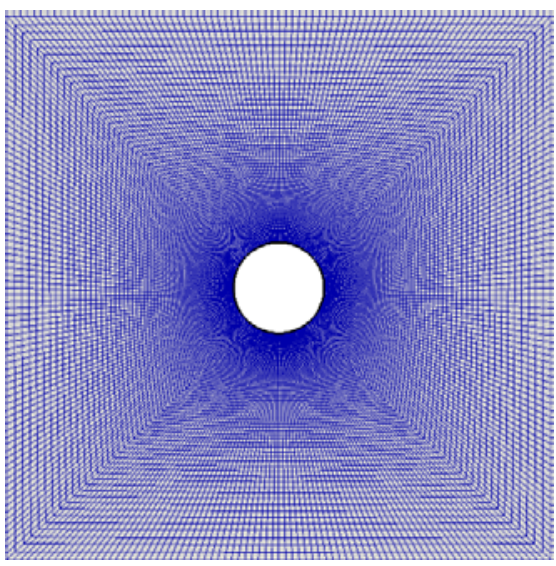

(B) close-up view of mesh near the cylinder

Fig. 2. Mesh of the computational domain.

compared with the experimental results. The solution field and boundary conditions are the same as in Fig. 1 , and the number of 80000 cells has been selected for the present numerical analysis. In Fig. 4, the velocity and pressure coefficients for the mesh reliability are compared with a number of experimental studies. A comparison of velocity and pressure profiles also shows that this model is in good agreement with the experimental results. Based on the conditions of the two-dimensional analysis and turbulence model, the difference in values in the frequency and amplitude of fluctuations is a maximum of $8 \%$. To ensure noise prediction, the numerical results are compared with the experimental study of Casino and Jacob (Casalino and Jacob 2003). As it was mentioned earlier, to predict the noise in this study, the two-dimensional solver with $k-\omega-s s t$ turbulence model is considered the same as previous studies. Due to the lower computational cost of two-dimensional models compared to 3D models (Cox et al. 1998, Khorrami et al. 2007, Liu et al. 2015, Liu et al. 2018 and Abbasi and Souri 2021b) and weaker noise estimation of the two-dimensional models, they are still more favorable for researchers (Ganta et al. 2019). In Fig. 5, it can be seen that the noise peak in numerical mode is $5 \mathrm{~dB}$ higher than in experimental mode. It has been pointed out that lift force 


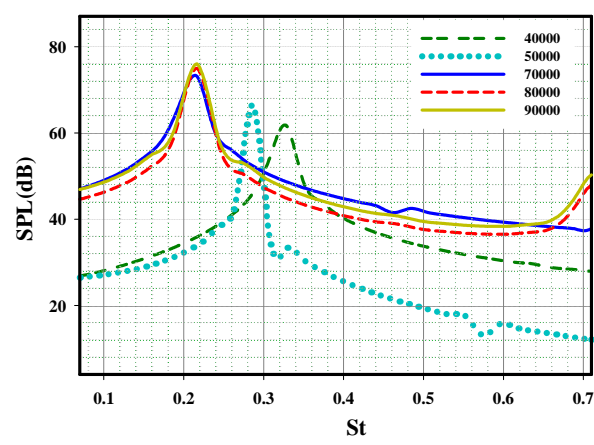

A) Sound pressure level results regarding the number of mesh cells for the baseline configuration.

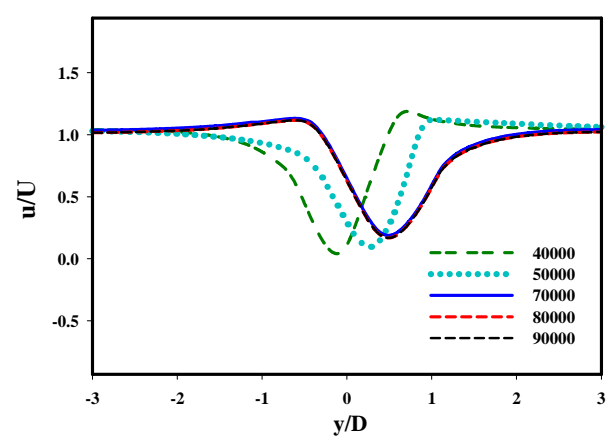

B) Cylinder wake profile independency results regarding the number of mesh cells for the baseline configuration $(x / D=2)$.

Fig. 3. Independence of results from mesh.

fluctuations are dominant in noise generation, but in two-dimensional models, lift force and consequently its fluctuations overestimate than experimental ones. Therefore, there may be a discrepancy between the numerical and experimental models. The predicted Strohal number is also 0.2167, while the experimental estimate is 0.20 . The discrepancy is due to the lack of consideration for the effect of spanwise. The SPL at another strouhal number may be different due to the URANS methodology not being able to represent smaller eddies in the near wake as well as a breakdown in the assumption of acoustic compactness.

\section{Results}

In this study, the role of different slot locations for blowing on aerodynamic noise and flow structure of a circular cylinder has been investigated. To investigate the effect of the blowing location, three different locations are considered behind the cylinder. All of them cover $10 \%$ of the cylinder circumference. By referring to Fig 6A, in (Case I), no blowing is applied. In case II, the locations of the blowing slot windows are defined between 0 to 18 deg and 342 to 360 deg angles. Case III locations are set between 36 to $54 \mathrm{deg}$ and 306 to 324 deg angles. Finally, case IV blowing slot windows locations are defined between 72 to $90 \mathrm{deg}$ and 270 to $288 \mathrm{deg}$ angles. For all cases, the ratio $V / U=0.25$ was employed for the blowing flow speed rate, where $\mathrm{V}$ is the blowing velocity in the angle perpendicular to the surface and $U$ is the freestream velocity $(20 \mathrm{~m} / \mathrm{s})$.

In Fig. $6 \mathrm{~B}$, the effect of the blowing location is indicated that the sound pressure level is plotted in terms of the Strouhal number. It is recognized that in the second case, the Noise spectra peak (NSP) is reduced approximately by $32 \mathrm{~dB}$. Also, the results indicated that by changing the blowing location to a far distance from the horizontal axis in case IV, the

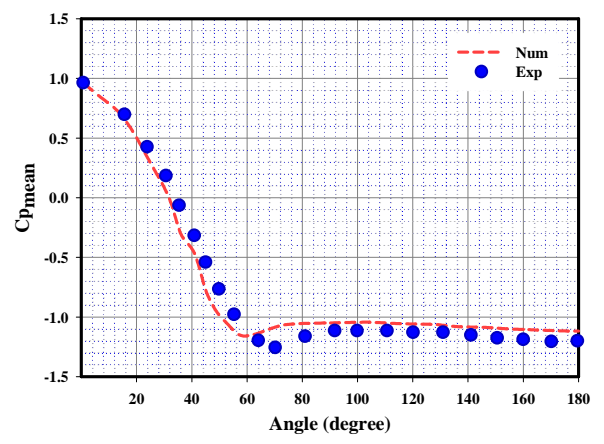

A) Mean pressure distributions around cylinders.

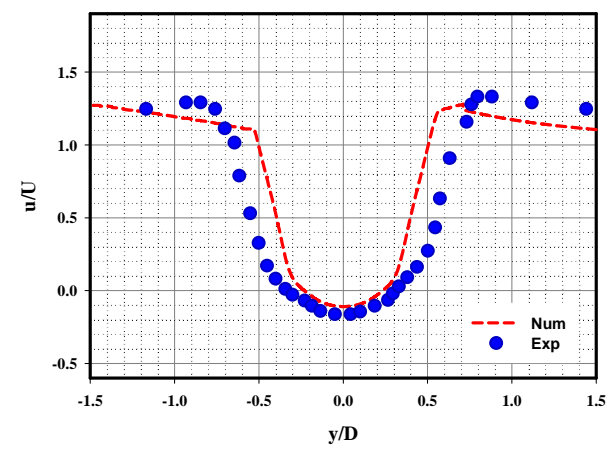

B) Profiles of the time-averaged streamwise velocity in the cylinder wake $(x / D=1)$

Fig. 4. Aerodynamic characteristics validation (numerical with the experimental (Gao et al. 2017)).

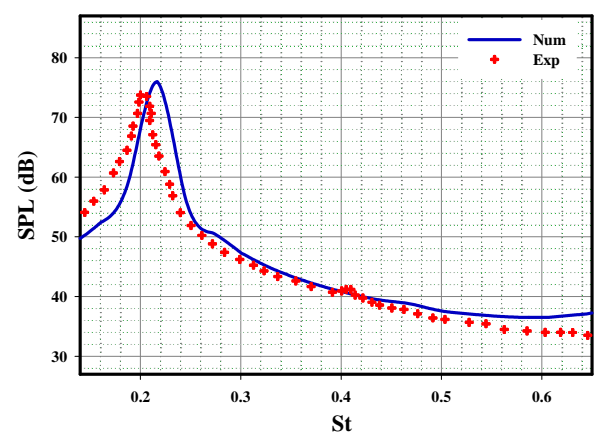

Fig. 5. Comparison of the predicted Sound Pressure Level (SPL) with the experimental (Casalino and Jacob 2003) results for a

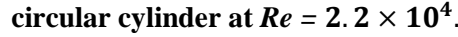



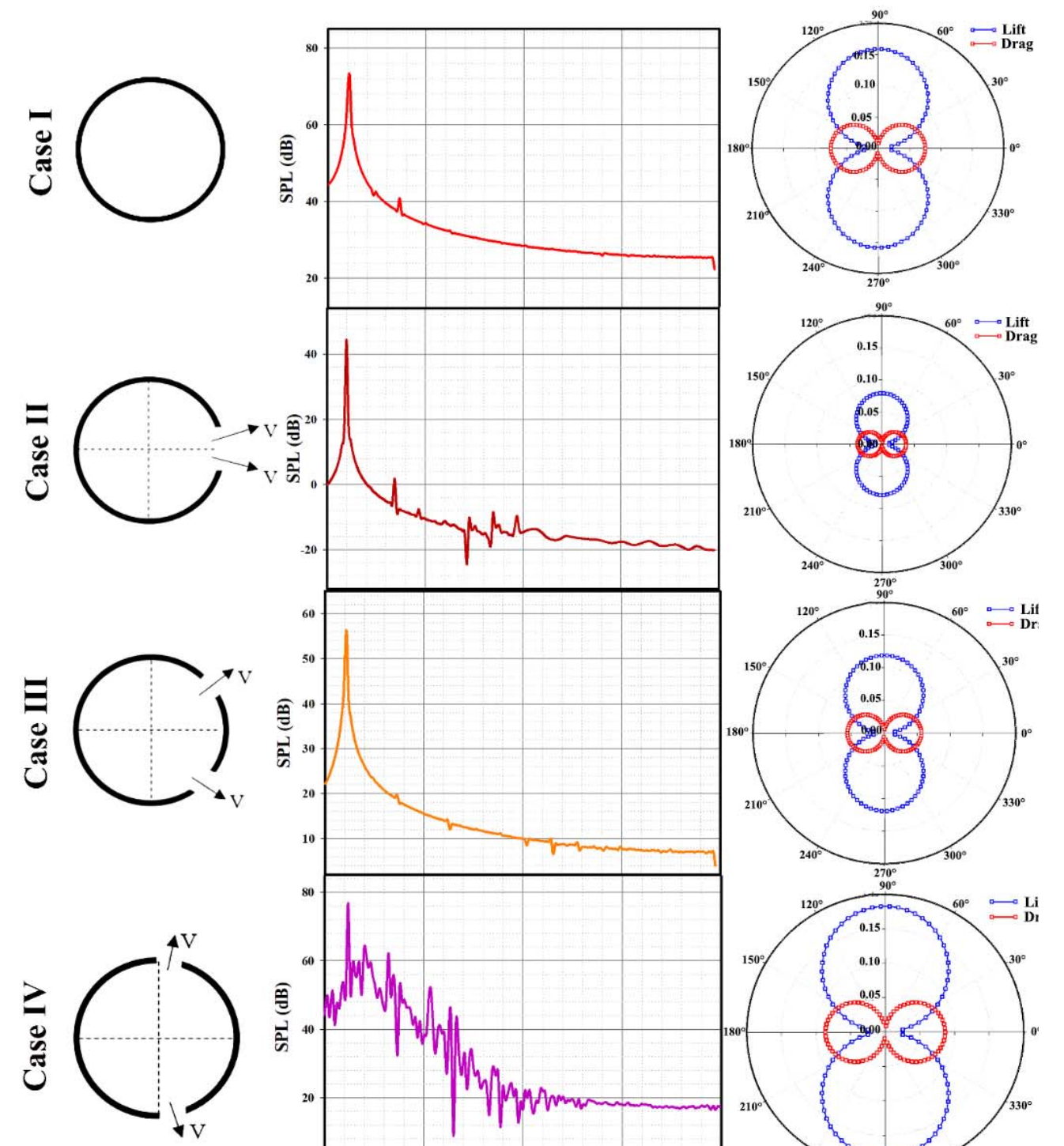

(A)

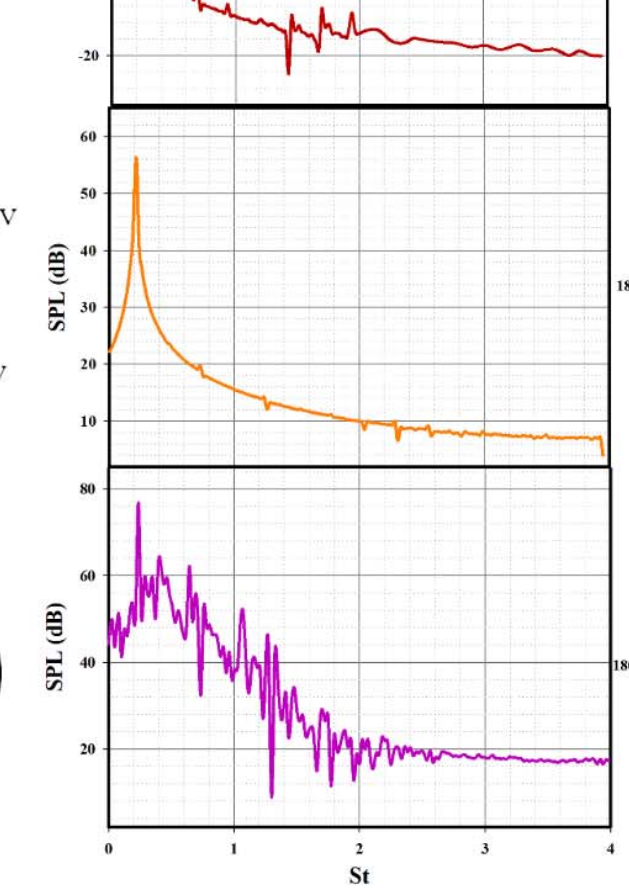

(B)
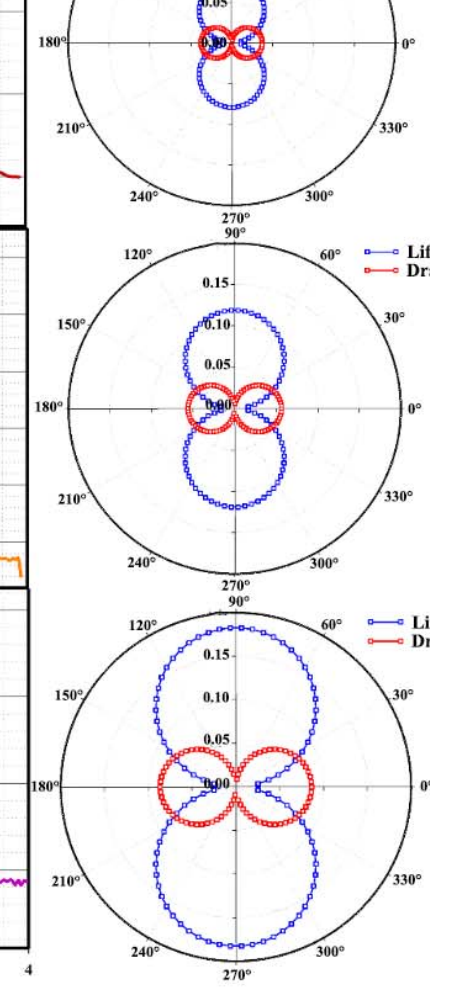

(C)

Fig. 6. (A) Schematic of blowing location in different cases, (B) Comparison of SPL for different blowing slot location, (C) Directivity of RMS of the acoustic pressures due to the fluctuation of lift and drag.

NSP is increased by $4 \mathrm{~dB}$ compared to the first case. Cases I, II, and III indicate that the first harmonic is the most effective factor as the second and third harmonics are weaker over time. But in case IV, the spectrum fluctuations have increased, that is due to increased turbulence and increased vortices. For a deeper understanding, the structure of the flow and vortex shedding will also be examined.

It has been pointed out that the forces exerted on the solid surface are the most important factors in the production and propagation of noise. In the present study, the most important forces on the surface are lift and drag forces. Figure 6C shows that the root mean square (RMS) of the acoustic pressures caused by lift and drag forces. Directivity analysis was performed on the microphone location. The nature of the graphs indicates that the noise is dipole, in which the lift force is more effective in producing noise. Because the largest unsteady pressure amplitude occurs on the top and bottom surfaces of the cylinder. The sound waves emitted by the lift force are in the $\pm 90^{\circ}$ direction, while the sound waves 
associated with the drag are in the $0^{\circ}$ and $180^{\circ}$ direction. An interesting finding in these plots is that mainly between $150^{\circ}-210^{\circ}$ and $330^{\circ}-30^{\circ}$, the amount of sound pressure caused by drag is much higher than the lift force, while beyond this range, the role of lift force in noise generation is greater. That is, in line with the Sun et al. (2017), if the microphone is placed in the indicated locations, the noise due to drag is predominant

To have a better understanding of the present results, the changes of NSP and Strouhal number in Fig. 7 are presented. Figure 7A shows that the lowest amount of noise recorded in case II which is in the horizontal direction with the flow. If the angle between the blowing and the flow is increased, the NSP increases as well. As in case $\mathrm{IV}$, where the blowing angle is perpendicular to the flow direction, the highest NSP value is recorded, which is higher than the reference value. Strouhal number changes in Fig. 7B shows that in the case where the lowest noise level is recorded (i.e case II), the strouhal number is also minimal, and Conversely, in the case where the highest noise level is recorded (i.e case IV) the value of the strouhal number is about $10 \%$ higher than the reference case I.

This relationship is related to the structure and characteristics of the flow, that will be discussed below.

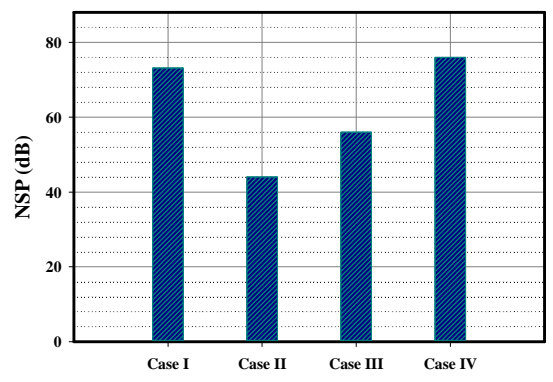

A) Noise spectra peak

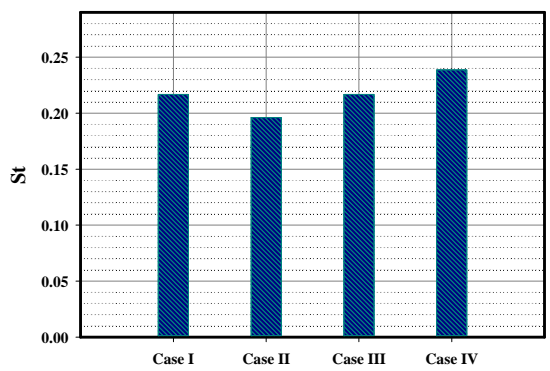

B) Strouhal number

Fig. 7. variations of the noise spectra peak value and the Strouhal number for different situations.

After supporting the effective role of the location of the blowing slot on the aerodynamic noise, it is better to examine the role of the intensity of the blowing, which changes by velocity $(0.05 \leq$ $V / U \leq 0.25)$.

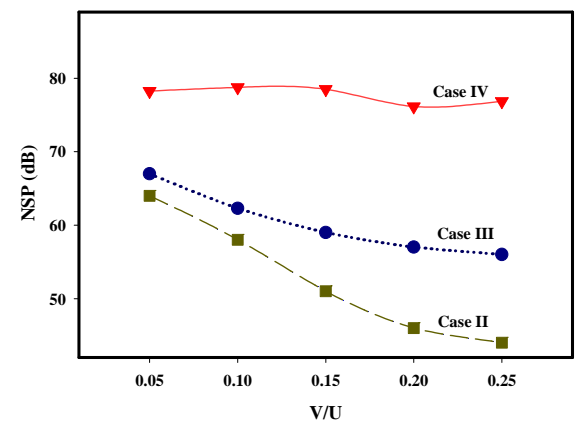

A) Noise spectra peak

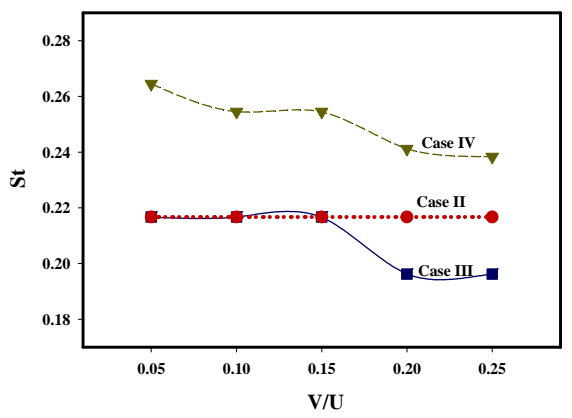

B) Strouhal number

Fig. 8. Variation of the NSP and the Strouhal number for different situations in different $V / U$ ratios.

changes in the NSP and Strouhal number for different blowing velocities for all cases are addressed in Fig. 8. Due to Fig. 8A, it's clear that increasing the velocity of the blowing reduces the aerodynamic noise, and reducing the strouhal number as well. Therefore, increasing velocity blowing has reduced the desired noise reduction. Due to the aero acoustic conditions, Case II with an intensity of 0.25 is the best option for the noise reduction. It can be understood that, generally, in case IV, by applying a blowing, not only the NSP is not reducing, but it is increased relative to the case I. Figure 8B shows that in case IV and case III, the Strouhal number variation is significant with the change in blowing velocity, but in cases II, change of the Strouhal number is negligible.

It was found that aerodynamic forces are the main sources of sound production, which in turn cause relatively strong fluctuations owing to the periodic nature of vortex Shedding. Fluctuations of the aerodynamic forces are effective in producing noise (Siozos-Rousoulis et al. 2017). Although the mean aerodynamic forces not involved in noise generation, are of particular importance due to structural damage. Therefore, the variation of these two parameters has been investigated in 0.25 
intensity for all cases. Since changes in aerodynamic forces are important relative to the reference case (case I). The main non-dimensional parameters are defined as follows:

$$
\begin{aligned}
& C_{L_{r m s}}=\frac{\left|C_{L_{r m s I}}\right|}{\left|C_{L_{r m s I=0}}\right|} \\
& C_{D_{\text {rms }}}=\frac{\left|C_{D_{\text {rms }} \mid}\right|}{\mid C_{D_{\text {rms I }=0} \mid}} \\
& C_{L_{\text {mean }}}=\frac{\left|C_{L_{\text {mean I }} \mid}\right|}{\mid C_{L_{\text {mean I }=0} \mid}} \\
& C_{D_{\text {mean }}}=\frac{\left|C_{D_{\text {mean }} \mid}\right|}{\left|C_{D_{\text {mean I }=0}}\right|}
\end{aligned}
$$

Based on the results of Figure 9A, 9B, it is clear that the mean lift-to-drag ratio $\left(\frac{C_{L_{\text {mean }}}}{C_{D_{\text {mean }}}}\right)$ in cases II and III are higher than in other cases. Therefore, from an aerodynamic view, they are more appropriate.

This result is desirable in terms of aerodynamic coefficients. But in terms of aerodynamic noise, the results obtained in case III are not desirable. Also, the results obtained in case IV are unfavorable from both the aerodynamic coefficients and the noise caused by the flow. In this case, the destructive forces have greatly increased. The fluctuations of the aerodynamic

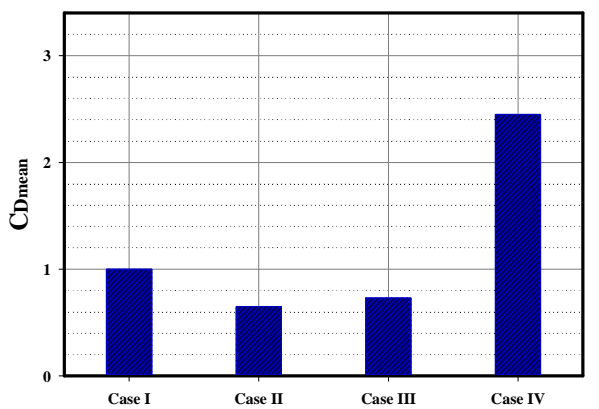

A) Mean Drag coefficient

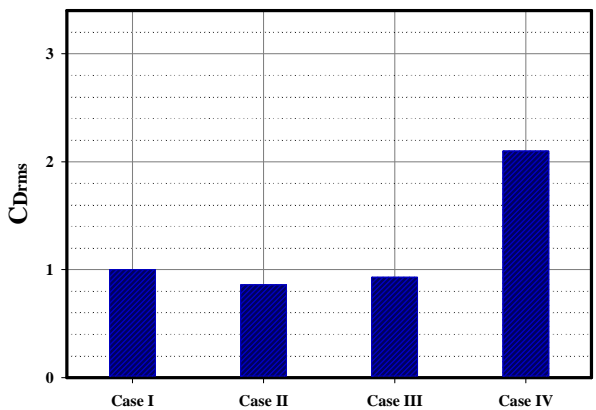

C) Root-mean-square Drag coefficient force coefficients associated with the aerodynamic noise are shown in Fig. 9C and 9D. The pattern of changes in the fluctuations of these coefficients is alike the changes in noise (Fig. 7A). Also, in case II when the aerodynamic noise has reached the lowest level, the drag and lift fluctuations are minimal.

Based on Fig 9.D. it is clear that due to the fact that the lift coefficient has a significant effect on the amount of sound production, in order to reduce noise, methods should be used that reduce the amount of lift force. In the case IV, the elevator and drag fluctuations have increased by more than $150 \%$ and $200 \%$, respectively. These results well accord with the relationship between lift and drag coefficients with SPL.

Also, due to the effective role of lift coefficient fluctuations in noise generation and the destructiveness of the mean drag, changes in these two parameters are considered in different cases where the intensity of the blowing varies. Increasing the blowing intensity in all blowing slot location reduces the lift fluctuations. But, the lift fluctuations of case IV are still more than four times that of the other cases (Fig. 10A). Moderate drag changes in Fig. 10B suggested that for cases II and III, changing the intensity of the blowing does not have a significant effect on drag changes, but with increasing blowing intensity in case IV, the mean drag increases significantly.

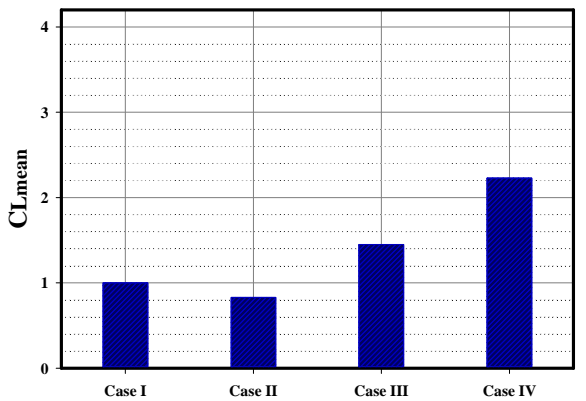

B) Mean lift coefficient

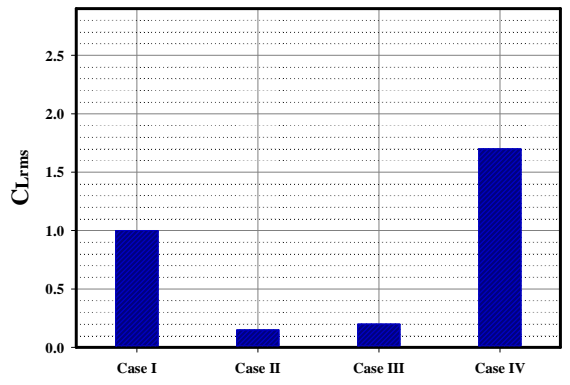

D) Root-mean-square lift coefficient

Fig. 9. Mean and Root-mean-square coefficients of lift and drag in terms of different cases, A) Mean Drag coefficient, B) Mean lift coefficient, C) Root-mean-square Drag coefficient, and D) Root-meansquare lift coefficient. 


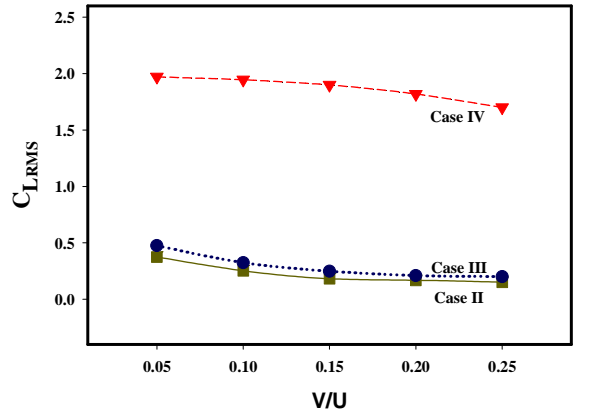

A) Root-mean-square lift coefficient

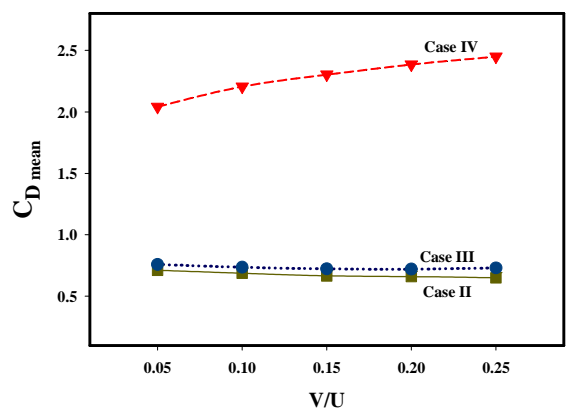

B) Mean Drag coefficient

Fig. 10. Mean drag coefficient and root mean square lift coefficients for different cases with different $V / U$ ratios.

The upstream boundary layer of the cylinder at the separation point overcomes the pressure gradient and it leads to the formation of shear layers in the vortex shedding. The vortex shedding behind the cylinder is caused by the interference of the opposing-sign shear-layers coming out of both sides of the cylinder. The shear layers separated behind the cylinder further grow due to the instability of vortices, that finally turn into the von Kármán vortex street. Figure 11. shows vorticity contours for the different blowing slot location (intensity is 0.25 ). The distribution of positive vorticity (counterclockwise rotation) and negative vorticity (clockwise rotation) are approximately symmetric relative to the axis of symmetry. Vorticity remains compressed and close to the cylinder for the case I and small vortices are generated. When the blowing is applied behind the cylinder and near the horizontal axis, it reduces the formation of vortices in the downstream of the cylinder. Applying the blowing in the appropriate location on the back of the cylinder causes the free shear layers to move downstream before roll-up occurs. Therefore, the due time for the formation of vortices increases, which leads to reducing the number of vortices generated. Decreasing the number of vortices, results in decreasing aerodynamic forces and their fluctuations cause to decreasing noise as well. The results that can be extracted from these Figs are the same as in Figs. 6 and 9. Applying blowing in the back of the cylinder surface, the vortex shedding phenomena are altered due to vortex roll-up distance. The occurrence of vortices and vortex roll-up distance determines the Strouhal number. Due to the vortices created in time $4 \mathrm{~T}$ for different cases, in case II, shear layers elongated and moved to downstream the cylinder and interact afterward. As a result, the vortex shedding slows down and the Strouhal number reduces. But in case IV contrary to what happens in case II, small vortexes are created on the back of the cylinder which result in a dramatic increase in the Strouhal number.

Mean pressure contour is significant as it determines the aerodynamic coefficients such as mean lift and drag. Since the drag coefficient is applied to the bluff body along the direction of flow, the difference between the upstream and downstream pressures of the cylinder determines the mean pressure drag value.

According to Fig. 12A, it is clear that applying the blowing in cases II, and III increases the pressure in the wake region of the cylinder and reduces the pressure difference between the upstream and downstream of the cylinder relative to the reference case, which results in decreasing drag as shown in Fig. 9A. In case IV, the condition has changed dramatically, forming a widely lowpressure area at the downstream of the cylinder, resulting in a multiple increase in drag force relative to case I. However, due to the symmetry of the pressure distribution around the cylinder, the mean lift changes are not as much as the drag force. Although the results in Fig. 9B show an increase in the mean lift relative to the reference case.

Reynolds stress contours can indicate vortex generation, therefore decreasing stress in these contours indicates suppression of vortex. In these contours, there are two focal points with tension centers on the sides of the cylinder, which indicating the roll-up positions of the shear layers on both sides. Decreasing fluctuation intensity and the displacement of the focal points are indicative of the transfer of the shear layers to the downstream of the cylinder. It can be attributed to the forces and, consequently, to the radiation of sound pressures as well. According to Fig. 12B, the application of the blowing in cases II and III has caused the transfer of focal point to the downstream, which in case II has the least amount. However, in case IV, not only are the focal points closer to the solid, but they are also more intense.

Pressure fluctuations is the most important factor in noise generation, and are created mainly in the region of the near wake associated with vortex formation. Also, pressure fluctuations are created at the solid surface, in which case they support dipole sources (Curle 1955). The contour of rootmean-square (RMS) pressure distribution about the cylinder is shown in Fig. 12C. For the case I, high-pressure fluctuations are observed. The generated vortex shedding and its fluctuations impact a wide zone of the flow behind the cylinder and on the cylinder surface as well. In cases, II and III, the application of blowing reduces pressure fluctuations. In case II, a very small zone with high 

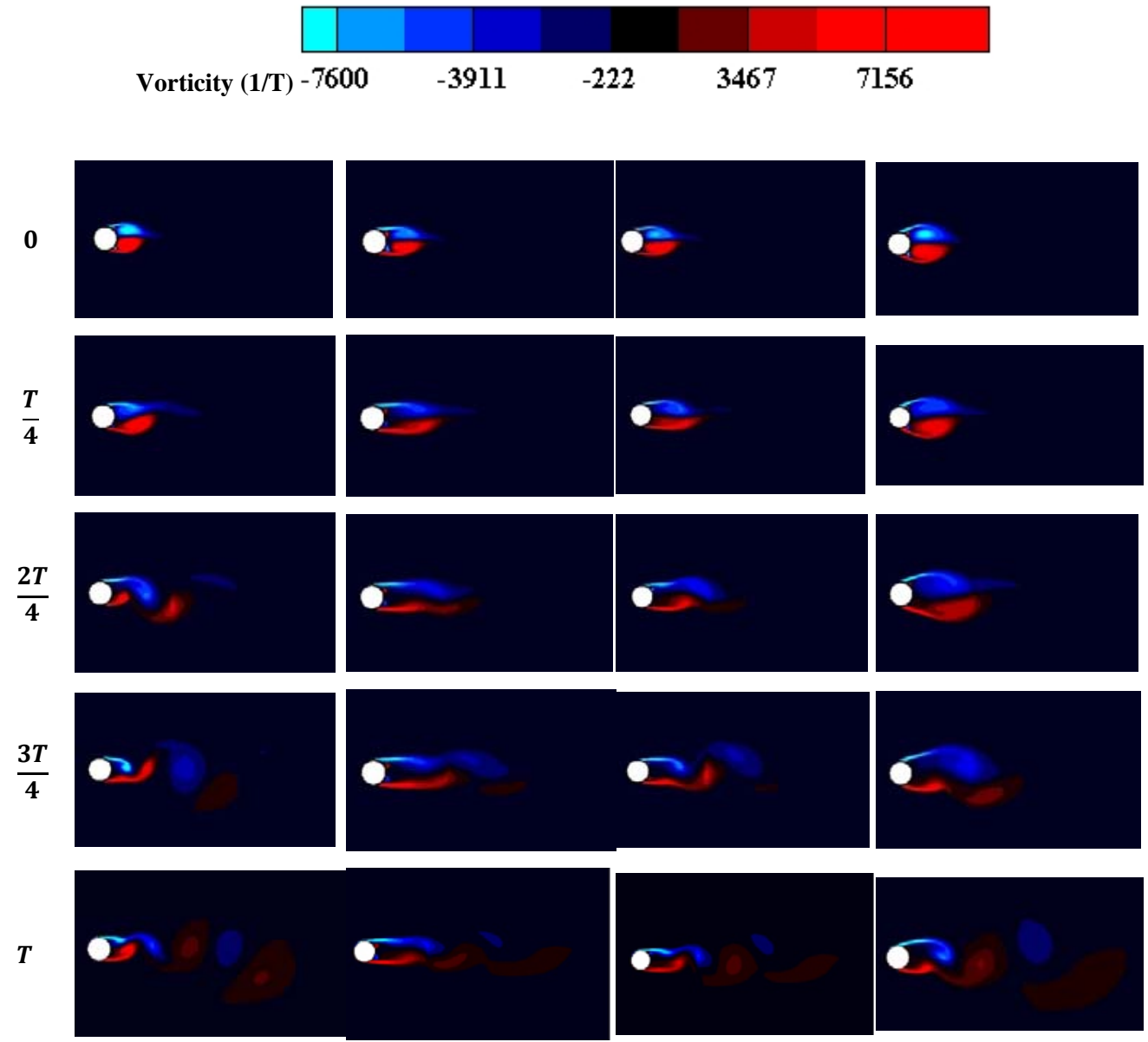

$4 T$
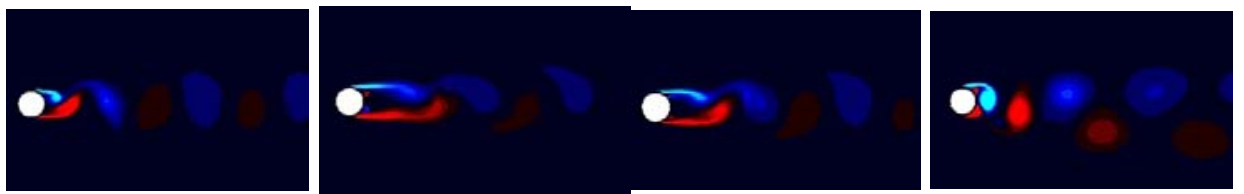

Case I

Case II

Case III

Case IV

Fig.11. Instantaneous spanwise vorticity $\left(\omega_{z}\right)$ contours for different cases ( $T$ is the period of vortex shedding).

fluctuation is observed. Therefore, the initial value is about 90 and it reaches less than 30 , which indicates a more than $68 \%$ decrease in fluctuations. The unsteady pressure amplitude created on the top and bottom surfaces are also reduced. In these two cases, the focal points of $P_{R M S}$ are not close to the solid surface, consequently, they do not have significant effects on noise generation. In other words, they reduce the oscillations of the pressure of the cylinder surface, which results in reducing noise. But in the case IV, the unsteady pressure covers the cylinder surface and the wide-area at the downstream of the cylinder, indicated an increase in fluctuations and turbulence, which in turn increases the oscillations of the aerodynamic forces and increases the propagation of sound waves from the sound source.

\section{Conclusion}

In this paper, a numerical study on the application of blowing through window slots on the cylinder surface as an active control method for aerodynamic noise was performed. The simulated aerodynamic noise was compared with experimental results and good agreement was observed. Three different blowing slot locations are considered, i.e., cases II (slots between 0-18 deg and 360-342 deg), III (slots between 36-54 deg and 306-324 deg) and IV (slots between 72-90 deg and 270-288 deg), respectively. The no-blowing 

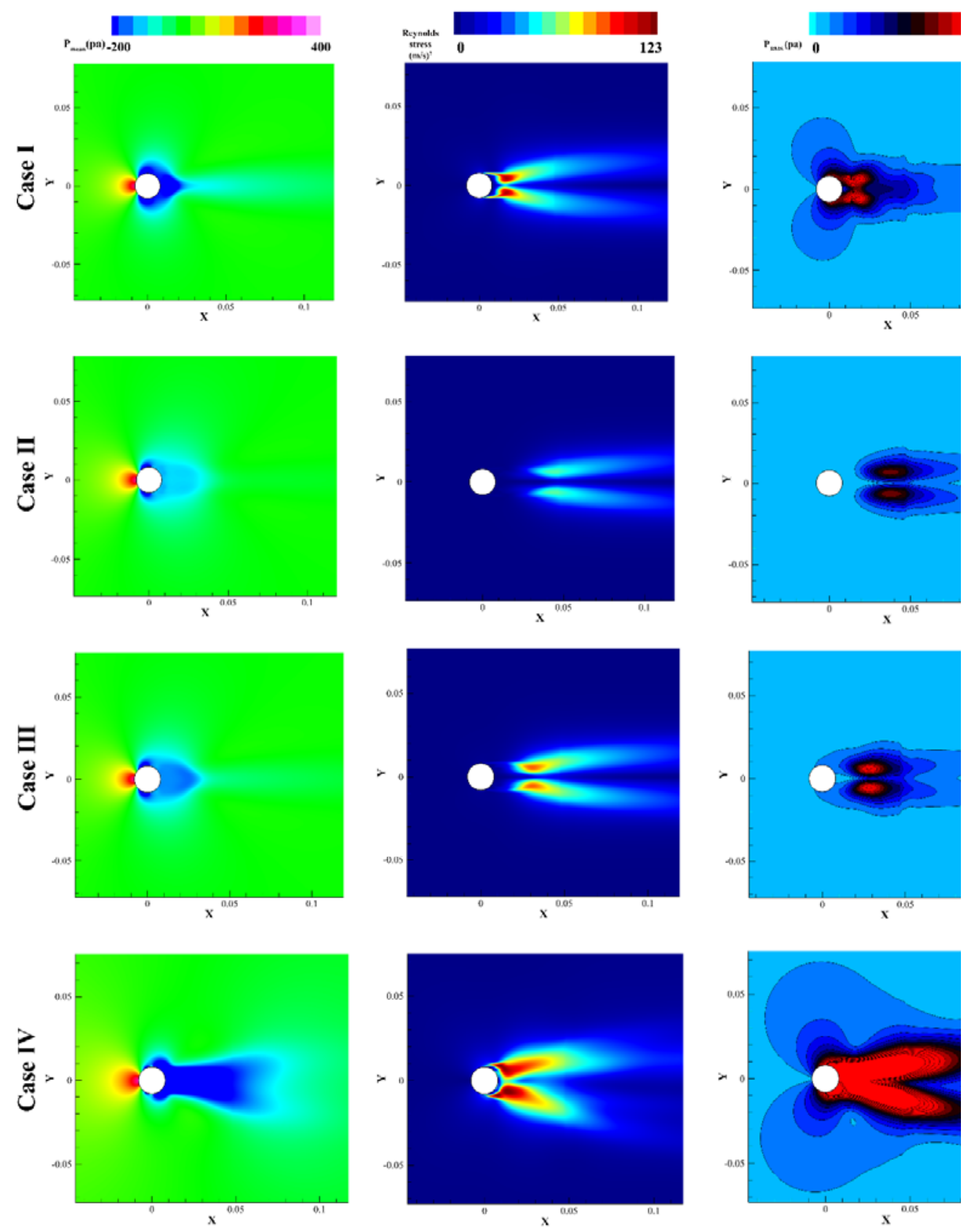

(A)

(B)

(C)

Fig. 12. (A) Mean static pressure for different cases, (B) Distribution of Reynolds shear stress, $<\boldsymbol{u}_{\boldsymbol{i}}^{\prime} \boldsymbol{u}_{j}^{\prime}$ $>$ for different cases, and (C) RMS pressure coefficient contours about the cylinder in turbulent flow for different cases.

case is regarded as case I. The main results obtained in this paper are summarized as follows:

$>$ The application of blowing near the horizontal axis of the cylinder, case II, showed the best performance in controlling the aerodynamic noise and the flow structure. This reduces the noise spectra peak value of approximately $32 \mathrm{~dB}$.
$>$ For locations far from the horizontal axis of the cylinder (i.e. case IV), blowing does not reduce the flow fluctuations and the aerodynamic noise as well. In case IV, the fluctuations are intensified which increases the noise intensity approximately by $4 \mathrm{~dB}$.

$>$ Changing the blowing location has a significant effect on the lift and drag coefficients. In case IV, these coefficients are 
doubled in comparison with the no-blowing case. In other words, the fluctuations of lift and drag coefficients, as well as their average values are increased, which is not desirable.

$>$ The application of blowing in case II has changed the vortex shedding pattern.

$>$ By increasing the blowing velocity in all cases, the sound intensity and the Strouhal number of the NSP are reduced due to a decrease in the flow fluctuations and weakening of the vortex shedding.

$>$ In cases, II and III, the increase in the blowing velocity reduces the drag and lift coefficients. Variations of lift fluctuations in case IV are similar to cases II and III. Increasing the blowing velocity, the lift coefficient is reduced. But its values are still larger than other cases. In case IV, the drag coefficient is increased by increasing the blowing velocity.

\section{Reference}

Abbasi, S. and M. Souri (2020). Reducing aerodynamic noise in a rod-airfoil using suction and blowing control method. International Journal of Applied Mechanics 12(04), 2050036.

Abbasi, S. and M. Souri (2021a). The Effect of Cylinder Blowing on Flow-induced Noise Reduction in a Cylinder-Airfoil. Amirkabir Journal of Mechanical Engineering 53(3 (Special Issue)), 7-7.

Abbasi, S. and M. Souri (2021b). On the passive control of aeroacoustics noise behind a square cylinder. Journal of the Brazilian Society of Mechanical Sciences and Engineering 43(2), 1-16.

Aradag, S., S. Siegel, J. Seidel, K. Cohen and T. McLaughlin (2011). Filtered POD-based low-dimensional modeling of the 3D turbulent flow behind a circular cylinder. International Journal for Numerical Methods in Fluids 66(1), 1-16.

Arcondoulis, E. J., T. F. Geyer and Y. Liu (2021). An investigation of wake flows produced by asymmetrically structured porous coated cylinders. Physics of Fluids 33(3), 037124.

Arcondoulis, E. J., Y. Liu, Z. Li, Y. Yang and Y. Wang (2019). Structured porous material design for passive flow and noise control of cylinders in uniform flow. Materials 12(18), 2905.

Assi, G. R. S., R. M. Orselli and M. Silva-Ortega (2019). Control of vortex shedding from a circular cylinder surrounded by eight rotating wake-control cylinders at $\mathrm{Re}=100$. Journal of Fluids and Structures 89, 13-24.

Bao, Z., G. Qin, W. He and Y. Wang (2018). Numerical investigation of flow around a slotted circular cylinder at low Reynolds number. Journal of Wind Engineering and Industrial Aerodynamics 183, 273-282.
Casalino, D. and M. Jacob (2003). Prediction of aerodynamic sound from circular rods via spanwise statistical modelling. Journal of Sound and Vibration 262(4), 815-844.

Chen, W., C. Ji, M. M. Alam, J. Williams and D. $\mathrm{Xu}$ (2020). Numerical simulations of flow past three circular cylinders in equilateraltriangular arrangements. Journal of Fluid Mechanics 891, A14.

Chong, W. T., W. K. Muzammil, K. H. Wong, C. T. Wang, M.G. wani, Y. J. Chu and S. C. Poh (2017). Cross axis wind turbine: Pushing the limit of wind turbine technology with complementary design. Applied Energy 207, 78-95.

Cox, J. S., K. S. Brentner and C. L. Rumsey (1998). Computation of vortex shedding and radiated sound for a circular cylinder: subcritical to transcritical Reynolds numbers. Theoretical and Computational Fluid Dynamics 12(4), 233-253.

Curle, N. (1955). The influence of solid boundaries upon aerodynamic sound. Proceedings of the Royal Society of London. Series A. Mathematical and Physical Sciences 231(1187), 505-514.

Feng, L. H., J. J. Wang and C. Pan (2011). Proper orthogonal decomposition analysis of vortex dynamics of a circular cylinder under synthetic jet control. Physics of Fluids 23(1), 014106.

Feng, L. H. and J. J. Wang (2010). Circular cylinder vortex-synchronization control with a synthetic jet positioned at the rear stagnation point. Journal of Fluid Mechanics 662, 232.

Ganta, N., B. Mahato and Y. G. Bhumkar (2019). Modulation of sound waves for flow past a rotary oscillating cylinder in a nonsynchronous region. Physics of Fluids 31(9): 096103.

Gao, Y., X. Qu, M. Zhao and L. Wang (2019). Three-dimensional numerical simulation on flow past three circular cylinders in an equilateral-triangular arrangement. Ocean Engineering 189, 106375.

Inoue, O., M. Mori and N.Hatakeyama (2003). Control of aeolian tones radiated from a circular cylinder in a uniform flow. Physics of Fluids, 15(6), 1424-1441.

Khorrami, M. R., M. M. Choudhari, D. P. Lockard, L. N. Jenkins and C. B. McGinley (2007). Unsteady flowfield around tandem cylinders as prototype component interaction in airframe noise. AIAA Journal 45(8), 19301941.

Li, Z., T. Tang, Y. Liu, E. J. Arcondoulis and Y. Yang (2020). Numerical study of aerodynamic and aeroacoustic characteristics of flow over porous coated cylinders: Effects 
of porous properties. Aerospace Science and Technology 105, 106042.

Lin, J. C., J. Towfighi and D. Rockwell (1995). Near-wake of a circular cylinder: control, by steady and unsteady surface injection. Journal of Fluids and Structures 9(6), 659669.

Liu, H., M. Azarpeyvand, J. Wei and Z. Qu (2015). Tandem cylinder aerodynamic sound control using porous coating. Journal of Sound and Vibration 334, 190-201.

Liu, H., Y. Wang, J. Wei and Z. Qu (2018). The importance of controlling the upstream body wake in tandem cylinders system for noise reduction. Proceedings of the Institution of Mechanical Engineers, Part G: Journal of Aerospace Engineering 232(3), 517-531.

Ma, R., Z. Liu, Zhang G., C. J. Doolan and D. J. Moreau (2019). Control of Aeolian tones from a circular cylinder using forced oscillation. Aerospace Science and Technology 94, 105370.

Mathelin, L., F. O. Bataille and A. Lallemand (2002). The effect of uniform blowing on the flow past a circular cylinder. Journal of Fluids Engineering 124(2), 452-464.

Norberg, C. (1987). Effects of Reynolds number and a low-intensity freestream turbulence on the flow around a circular cylinder. Chalmers University, Goteborg, Sweden, Technological Publications 87(2), 1-55.

Rocha, P. C., H. B. Rocha, F. M. Carneiro, M. V. da Silva and A. V. Bueno (2014). k- $\omega$ SST (shear stress transport) turbulence model calibration: A case study on a small scale horizontal axis wind turbine. Energy 65, 412418.

Samion, S. R. L., M. S. M. Ali, A. Abu, C. J. Doolan and R. Z. Y. Porteous (2016). Aerodynamic sound from a square cylinder with a downstream wedge. Aerospace Science and Technology 53, 85-94.

Schetz, J. A. and B. Nerney (1977). Turbulent boundary layer with injection and surface roughness. AIAA Journal 15(9), 1288-1294.

Schlichting, H. and K. Gersten (2016). Boundary-layer theory, Springer.

Seo, J. H. and Y. J. Moon (2007). Aerodynamic noise prediction for long-span bodies. Journal of Sound and Vibration 306(3-5), 564-579.

Siozos-Rousoulis, L., C. Lacor and G. Ghorbaniasl (2017). A flow control technique for noise reduction of a rod-airfoil configuration. Journal of Fluids and Structures 69, 293-307.

Souri, M. and A. Mojra (2021). A nexus between active and passive control methods for reduction of aerodynamic noise of circular cylinder. International Journal of Mechanical Sciences, 106446.

Souri, M., F. Moradi Kashkooli, M. Soltani and K. Raahemifar (2021). Effect of Upstream Side Flow of Wind Turbine on Aerodynamic Noise: Simulation Using Open-Loop Vibration in the Rod in Rod-Airfoil Configuration. Energies 14(4), 1170.

Sun, Y., R. J. Fattah and X. Zhang (2017). Airfoil Leading Edge Noise Predictions Using a Viscous Mean Flow. 24th International Congress on Sound and Vibration, ICSV 2017.

Williams, D. R., H. Mansy and C. Amato, (1992). The response and symmetry properties of a cylinder wake subjected to localized surface excitation. Journal of Fluid Mechanics 234, 71-96.

Williams, J. F. and D. L. Hawkings (1969). Sound generation by turbulence and surfaces in arbitrary motion. Philosophical Transactions for the Royal Society of London. Series A, Mathematical and Physical Sciences, 321342.

Zhang, J., W. Chu, H. Zhang, Y. Wu and X. Dong (2016). Numerical and experimental investigations of the unsteady aerodynamics and aero-acoustics characteristics of a backward curved blade centrifugal fan. Applied Acoustics 110, 256-267.

Zhang, P., Y. Liu, , Z. Li, H. Liu and Y. Yang, (2020). Numerical study on reducing aerodynamic drag and noise of circular cylinders with non-uniform porous coatings. Aerospace Science and Technology 107, 106308.

Zhdanov, V. L., S. A. Isaev and H. J. Niemann (2001). Control of the near wake of a circular cylinder in blowing out of low-head jets. Journal of Engineering Physics and Thermophysics 74(5), 1100-1103.

Zhu, H., G. Li and J. Wang (2020). Flow-induced vibration of a circular cylinder with splitter plates placed upstream and downstream individually and simultaneously. Applied Ocean Research 97, 102084.

Zhu, H. B., H. Ping, R. Wang, Y. Bao, D. Zhou and Z. L. Han (2019a). Flow-induced vibration of a flexible triangular cable at low Reynolds numbers. Physics of Fluids 31(5), 057101 .

Zhu, H., H. Zhao and T. Zhou (2019b). Direct numerical simulation of flow over a slotted cylinder at low Reynolds number. Applied Ocean Research 87, 9-25. 